\title{
KURZMIT'TEILUNGEN
}

\section{Untersuchungen zur Wirkung der Nikotinsäure auf die stimulierte Lipolyse}

\section{Studies on the action of nicotinic acid on stimulated lipolysis}

Von P. Schwandt und Th. Hartmann

Ais der I. Medizinischen Klinik der Universität München
(Direktor: Prof. Dr. H. Scbuviegk)

(Eingegangen am 22. Juni 1968)

Nikotinsäure ist in zahlreichen Untersuchungen zur Hemmung der Fettmobilisation verwandt worden; über die Angriffspunkte dieser Substanz im lipolytischen System liegen $z$. T. widersprüchliche Angaben vor. So halten CARLson und Bally (1) sowie BjörNTrop (2) eine Hemmung der Lipase durch Nikotinsäure genauso für möglich wie eine Begünstigung der Reveresterung. Stock und WestermanN (3) nehmen an, daß das lipolytische System durch Nikotinsäure lipasenäher blockiert wird als durch Sympathicolytica. Peterson und Mitarbeiter (4) konnten die durch Theophyllin induzierte Lipolyse mit Nikotinsäure kompetitiv hemmen und keine direkte Wirkung auf die Lipaseaktivität nachweisen; sie vermuten, daß Nikotinsäure über eine Veränderung des Spiegels von cyclischem AMP lipolysehemmend wirkt. KrISHNA und Mitarbeiter (5) konnten die Hemmwirkung von Nikotinsäure auf die durch Theophyllin induzierte Lipolyse durch Zugabe von cyclischem AMP aufheben und mit $10 \mathrm{nM}$ Nikotinsäure eine Aktivierung der Phosphodiesterase ${ }^{1}$ ) aus Fettgewebe nachweisen; sie nehmen an, daß Nikotinsäure zumindest teilweise über eine Aktivierung der Phosphodiesterase lipolysehemmend wirkt. Im Gegensatz dazu fanden KupIECKI und MARSHALL (6), daß Nikotinsäure in Konzentrationen von $10 \mu \mathrm{M}$ bis $10 \mathrm{~mm}$ keinen Einfluß auf die Phosphodiesterase des Fettgewebes hat. Wir haben untersucht, ob Nikotinsäure die lipolytische Wirkung von cyclischem AMP hemmt und ob sich mit Theophyllin der Hemmeffekt von Nikotinsäure auf die mit ACTH stimulierte Lipolyse aufheben läßt.

\section{Methodik}

Die Untersuchungen wurden in vitro am epididymalen Fettgewebe von etwa $300 \mathrm{~g}$ schweren Wistar-Ratten (mit AltrominPellets ad libitum gefüttert) entsprechend früheren Angaben (7) durchgeführt. In einer Versuchsreihe wurden pro Inkubationsansatz $(3,0 \mathrm{~m} l) 1 \mathrm{mMol}$ Dibutyryl-A-3',5'-MP (Fa. Boehringer, Mannheim) und 5, 50, 100 und $1000 \mu \mathrm{g}$ Nikotinsäure (Fa. Merck Darmstadt) zugesetzt. Bei den übrigen Versuchen waren im Ansatz folgende Substanzen enthalten: 0,03 I. E. ACTH (Fa. Schering, Berlin), $1 \mathrm{mMol}$ Theophyllin ( $\mathrm{Fa}$. Homburg-Chemie, Frankfurt) und $1 \mathrm{mMol}$ Nikotinsäure. Bei einem Teil dieser Versuche wurden Theophyllin und Nikotinsäure bei Inkubationsbeginn zusammen zugesetzt und bei einem anderen Teil getrennt, d. h. zu Beginn der Inkubation und $1 \mathrm{Std}$. danach. Die freien Fettsäuren im Inkubationsmedium wurden einheitlich nach 3stdg. Inkubationsdauer bestimmt.

\section{Ergebnisse}

Tabelle 1 zeigt, daß die mit $1 \mathrm{mMol}$ Dibutyryl-A-3', $5^{\prime}-\mathrm{MP}$ von $1,56 \pm 0,74 \mathrm{mVal} / \mathrm{l}$ auf $4,71 \pm 1,43 \mathrm{mVal} / l$ stimulierte Freisetzung freier Fettsäuren auch mit hohen Konzentrationen von Nikotinsäure nicht zu hemmen war.

Die durch ACTH stimulierte Lipolyse war nach 3stdg. Inkubation mit Nikotinsäure um $29 \%$ gehemmt; im gleichen Zeitraum ließ sich die ACTH induzierte Fettsäurefreisetzung mit Theophyllin um 19\% steigern. Wurden dagegen zu Inkubationsbeginn

1) Phosphodiesterase $=$ Orthophosphorsäurediester Phosphohydrolase (EC 3.1.4.1).
Tab, 1

Einfluß verschiedener Nikotinsäurekonzentrationen auf die mit $1 \mathrm{mMol}$ Dibutyryl-A-3',5'-MP/Ansatz stimulierte Lipolyse in vitro. Die Mengenangaben beziehen sich auf $3 \mathrm{ml}$ Inkubationsmedium. Die freien Fettsäuren sind in mVal/l Inkubationsmedium/250 mg Fettgewebe/3 Stdn. angegeben

\begin{tabular}{ccc}
\hline Nikotinsäure $(\mu \mathrm{g} / 3 \mathrm{ml})$ & $\mathrm{n}$ & freie Fettsäuren \\
\hline 0 & 16 & $4,71 \pm 1,43$ \\
5 & 10 & $4,56 \pm 0,85$ \\
50 & 6 & $4,86 \pm 0,44$ \\
100 & 10 & $4,83 \pm 1,01$ \\
1000 & 8 & $4,69 \pm 1,04$ \\
\hline
\end{tabular}

Theophyllin und Nikotinsäure in äquimolaren Konzentrationen zugegeben, betrug die Hemmung der mit ACTH stimulierten Lipolyse nur $5 \%$.

In Abbildung 1 sind die Differenzwerte der freien Fettsäuren gegenüber der Spontanlipolyse bei Zusatz von Theophyllin und Nikotinsäure in verschiedener Reihenfolge zu dem mit ACTH inkubierten Fettgewebe dargestellt. Wurde zuerst Theophyllin zugesetzt, so war durch Nikotinsäurezusatz nach 1 Std. keine

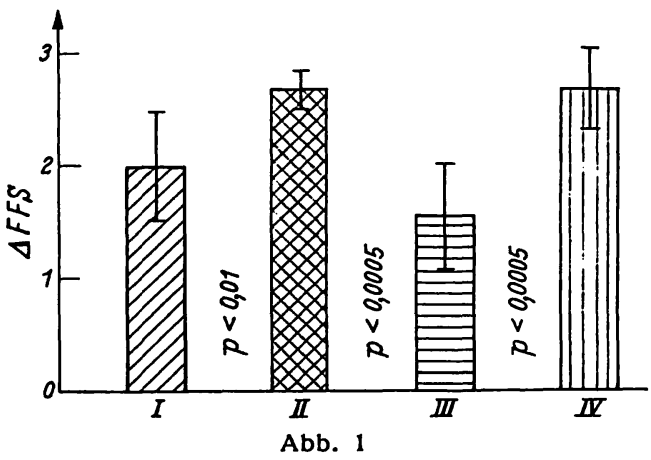

Einfluß von Theophyllin und Nikotinsäure auf die mit ACTH stiEinfluß von Theophy mulierte Lipolyse in vitro

Die Differenzwerte der freien Fettsäuren ( $\triangle \mathrm{FFS}$ ) gegenüber der Spontanlipolyse sind in mVal/l Inkubationsmedium/250 mg Fettgewebe/3 Stdn. angegeben

I 0,03 I. E. ACTH

II 0,03 I. E. ACTH $1 \mathrm{mMol}$ Theophyllin

III 0,03 I. E. ACTH $1 \mathrm{mMOl}$ Nikotinsäure; nach $1 \mathrm{Std}, 1 \mathrm{mMol}$ Theophyllin

IV 0,03 I. E. ACTH $1 \mathrm{mMol}$ Theophyllin; nach $1 \mathrm{Std}$. $1 \mathrm{mMol}$ Nikotinsäure

Alle Mengenangaben beziehen sich auf $3 \mathrm{ml}$ Inkubationsmedium.

signifikante Änderung $(2,70 \pm 0,38 \mathrm{mVal} / l ; \mathrm{n}=6)$ gegenüber der mit Theophyllin und ACTH stimulierten Lipolyse $(2,69 \pm$ $0,18 \mathrm{mVal} / l ; \mathrm{n}=6$ ) zu beobachten. Im Vergleich zu der nur durch ACTH induzierten Fettsäurefreisetzung $(1,99 \pm 0,49 \mathrm{mVal} /)$ fanden sich hingegen immer noch signifikant höhere Werte freier Fettsäuren $(p<0,025)$. - Bei Zusatz von Nikotinsäure zu Beginn der Inkubation und von Theophyllin nach $1 \mathrm{Std}$. war die mit ACTH und Theophyllin stimulierte Lipolyse signifikant gehemmt $(1,55 \pm 0,49 \mathrm{mVal} / l ; \mathrm{n}=9 ; \mathrm{p}<0,005)$; der Unterschied $\mathrm{zu}$ der nur mit ACTH stimulierten Freisetzung freier Fettsäuren war dagegen nicht mehr signifikant $(p<0,15)$.

\section{Diskussion}

Nach den gegenwärtigen Vorstellungen über das Lipolysesystem (8) sind drei Angriffspunkte für die Hemmwirkung der Nikotinsäure denkbar:

1. Hemmung der Bildung von cyclischem $3^{\prime}, 5^{\prime}$-AMP über die Adenylcyclase,

2. Stimulierung der Phosphodiesterase,

3. Hemmung der hormonempfindlichen Triglyceridlipase.

Gegen die erstgenannte Möglichkeit sprechen unsere Befunde, nach denen durch Zusatz von Theophyllin die Hemmwirkung von Nikotinsäure auf die mit ACTH stimulierte Lipolyse annähernd aufgehoben wird; dabei besteht kein Unterschied, ob Nikotinsäure und Theophyllin gleich bei Inkubationsbeginn zugesetzt werden oder ob Theophyllin erst 1 Std. später zugegeben wird. ACTH erhöht 
bekanntlich über eine Stimulierung der Adenylcyclase den Spiegel von cyclischem 3',5'-AMP; Theophyllin hemmt den Abbau dieses cyclischen Monophosphats über eine Blockierung der Phosphodiesterase (8). Nimmt man eine Hemmung der Adenylcyclase durch Nikotinsäure an, dann müßte auch nach Zusatz von Theophyllin der AMP-Spiegel erniedrigt bleiben, da ja Theophyllin nicht zu einer vermehrten Bildung von 3',5'-AMP führt; das ist nach unseren Befunden nicht der Fall.

Eine Stimulierung der Phosphodiesterase durch Nikotinsäure erscheint aufgrund des Befundes einer fehlenden Hemmwirkung von Nikotinsäure auf die mit Dibutyryl-A-3', $5^{\prime}-\mathrm{MP}$ stimulierte Lipolyse zunächst ebenfalls unwahrscheinlich. Es ist jedoch bekannt, daß Dibutyryl-A-3',5'-MP von der Phosphodiesterase nicht abgebaut wird (9). Das Acylderivat haben wir in Ubereinstimmung mit anderen Autoren (9-11) deshalb zur Stimulierung der Lipolyse verwandt, weil cyclisches 3',5'-AMP wegen seiner schlechten Membrangängigkeit kaum lipolytisch wirkt (9). Die fehlende Wirkung von Nikotinsäute auf die mit Dibutyryl-A-3',5'MP stimulierte Lipolyse spricht also keineswegs gegen die Annahme von KrishNa und Mitarbeitern (5). Für eine Stimulierung der Phosphodiesterase durch Nikotinsäure könnte die Tatsache herangezogen werden, daß die mit ACTH und Theophyllin stimulierte Fettsäurefreisetzung durch Zugabe von Nikotinsäure nach 1 Std. nicht gehemmt wird und $\mathrm{da} ß$ die hierbei gemessenen Fettsäurewerte sogar noch über denen bei der mit ACTH stimulierten Lipolyse liegen: die Phosphodiesterase ist offenbar durch Theophyllin so weitgehend blockiert, daß Nikotinsäure nicht in der Lage ist, dieses $3^{\prime}, 5^{\prime}$-AMP abbauende Enzym ausreichend zu reaktivieren.

Die dritte Möglichkeit - Hemmung der hormonempfindlichen Triglyceridlipase - scheidet deshalb aus, weil sich die Hemmwirkung von Nikotinsäure mit Theophyllin aufheben läßt. $\mathrm{Zu}$ dem gleichen Ergebnis kommen Peterson und Mitarbeiter (4), die keine Nikotinsäurewirkung auf die hormonempfindliche Lipase nachweisen konnten.

Zusammenfassend läßt sich sagen, daß aufgrund der z. Z. gültigen Vorstellungen über das lipolytische System eine Stimulierung der Phosphodiesterase durch Nikotinsäure als mögliche Erklärung für die Hemmwirkung dieser Substanz auf die hormonstimulierte Lipolyse diskutiert werden kann.

\section{Literatur}

1. Carlson, L. A. und P. R. Bally, In: Handbook of Physiology, Section V, Adipose Tissue, p. 557. Ed. E. A. Renold und C. F. Cahrll. Amer. Physiol. Soc., Washington (1965). - 2. BJöRNTORP, P., Metabolism 14, 836 (1965). - 3. Stock, K. und E. Westermann, Naunyn-Schmiedebergs Arch. exp. Pathol. Pharmakol. 254, 334 (1966). - 4. Peterson, M. J., C. C. Hillman und J. Ashmore, Federation Proc. 26, 400 (1967). - 5. Krishina, G., B. Weiss, J. I. Davies und S. Hynie, Federation Proc. 25, 719 (1966). - 6. Kupiecki, F. P. und N. B. Marshall, J. Phaímacol. Exper. Therap. Baltimore 160, 166 (1968). - 7. ScHwandr, P., M. KNedel und R. Lindlbauer, diese Z. 6, 81 (1968). 8. Sutherland, E. W. und R. W. Butcher, In: Protein and Polypeptid Hormones, S. 141, Ep. M. Margoulies. Internat. Congr. Series No. 161, Excerpta Medica Foundation, Amsterdam-New York-London-Paris-Milan-Tokyo-Buenos Aires (1968). 9. Butcher, R. W., R. J. Ho, H. C. Meng und E. W. SutherLAND, J. biol. Chemistry 240, 4515 (1965). - 10. BraY, G. A., Biochem. Biophys. Res. Commun. 28, 621 (1967). - 11. Aurich, A., K. Stock und E. WestermanN, Life Sciences 6, 929 (1967).

Dr. P. Schwandt I. Med. Univ. Klinik 8 München 15 Ziemssenstraße 1

\section{Spektrophotometrische Messung} von Porphyrinmethylester-Cu-Chelaten

Spectrophotometric measurement of porpbyrin metbyl ester-Cu chelates

Von M. Doss

Aus dem Hygiene-Institut der Universität Marburg a. d. Labn (Direktor: Prof. Dr. R. Siegert)

(Eingegangen am 25. Juni 1968)

Úber eine dünnschichtchromatographische Methode zur Isolierung und Bestimmung der Porphyrine als Methylester wurde vor kurzem berichtet $(1,2)$. Die Auftrennung erfolgte an KieselgelH-Platten in Lösungsmittelsystemen, die sich aus Benzol, Essigsäureäthylester, Butanol und Methanol zusammensetzen (1). Aus verschiedenem biologischen Material, wie Urin $(1,2)$, Erythrocyten $(3,4)$ und Bakterien (5), konnten chromatographisch reine Substanzen erhalten werden. Eine Rechromatographie ließ sich oft nicht umgehen, wenn kleine Porphyrinmengen mit größeren Anteilen anderer Chromogene im Chloroformextrakt vorlagen (2). Beimischungen von Lipoiden wie Glyceride, Glycerinphosphatide, Fettsäuren und ihre Methylester sowie Cholesterin und Cholesterinester haben keinen Einfluß auf die Bestimmung der Porphyrine (6).

Bei Versuchen, die analytische Empfindlichkeit der Methode zu verbessern, zeigte sich, daß Porphyrinmethylester innerhalb von 15 Min. mit einer Lösung von Kupfer(II)-acetat $(0,05 \%$, w/v) in Chloroform-Methanol $(1: 1, \nabla / v)$ Chelate bilden, die über Wochen stabil bleiben. Nach Bildung der Chelate ist es nicht mehr erforderlich, die Proben vor Licht zu schützen $(1,7)$. Der Extinktionskoeffizient der einzelnen Porphyrinmethylester an der SoretBande erhöht sich bei der Cu-Chelatbildung um 60 bis $100 \%$ gegenüber der Registrierung in reinem Chloroform. Die Empfindlichkeit des spektrofluorometrischen Nachweisbereiches wird mit dem Porphyrinmethylester-Cu-Komplex jedoch nicht erreicht. Ein wesentlicher Vorteil der Messung besteht darin, daß die schmalbasige und spitze Absorptionsbande der Prophyrinmethylester-Cu-Chelate bei $400 \mathrm{~nm}$ (Abb.) durch Beimischungen anderer

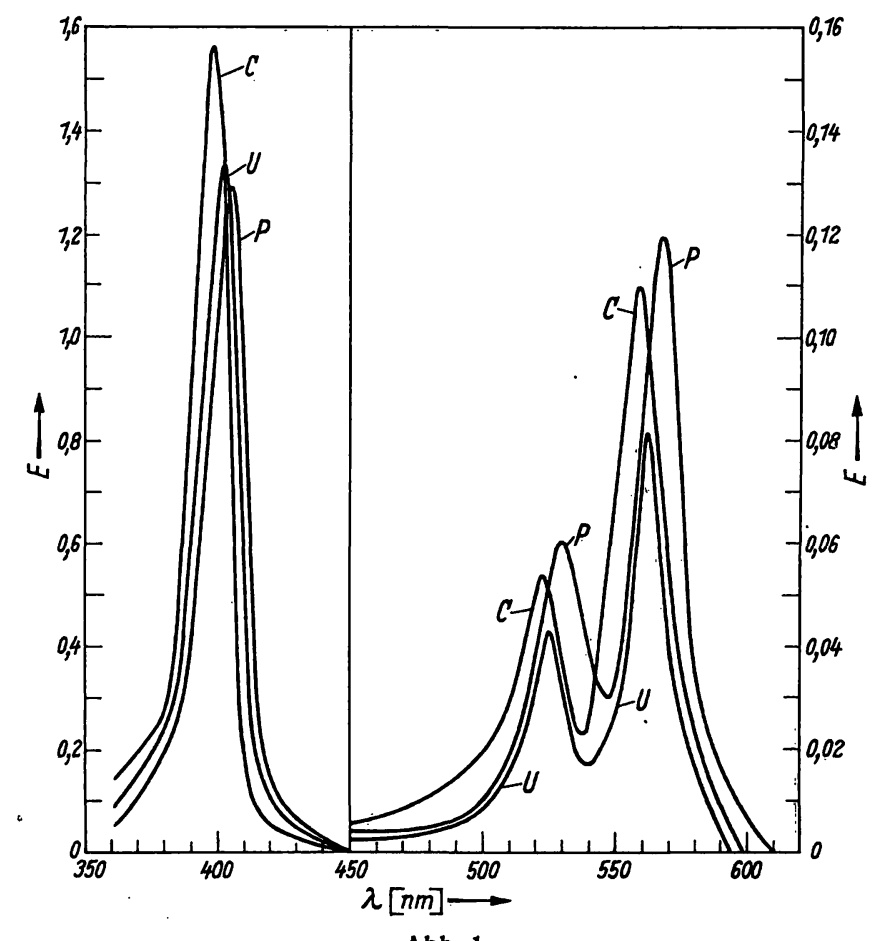

Abb. 1

Absorptionsspektren der $\mathrm{Cu}$-Chelate von

(P) Protoporphyrin-IX-dimethylester (4,96 $\mu \mathrm{Ms})$ aus Erythrocyten (C) Koproporphyrin-III-tetramethylester $(4,11 \mu \mathrm{M})$ von Achromobacer

(U) Uroporphyrin-III-oktamethylester $(3,37 \mu \mathrm{M})$ aus Urin. Absorptionsmaxima vgl. Tab. Spektrophotometer "Cary M 15“. 
Substanzen (Chromogene) weniger störbar ist als die SoretBande der Porphyrinmethylester in organischen Lösungsmitteln $(1,2,7)$. Damit wird eine Rechromatographie (2) zur Isolierung der Porphyrinmethylester in reinster Form weitgehend überflüssig.

\section{Methodik \\ Vorbereitung zur Bestimmung der Porply rine als Methylester aus bio- logischem Material}

Die Porphyrine werden, wie beschrieben $(2,3)$, in gefriergetrocknetem Material zu Porphyrinmethylestern verestert und extrahiert. Für die Aufarbeitung einer Reihe von Proben (Leberzellkulturen (8), Erythrocyten aus 1 bis $2 \mathrm{~m} l$ Vollblut, Bakterien bis zu $20 \mathrm{mg}$ Trockengewicht und bakterielle zellfreie Systeme mit einem Proteingehalt von 3 bis $8 \mathrm{mg}$ (5)) wird ein Reagenzgefäß mit Schliff (9) verwendet. Gelangen größere Volumina zur Analyse, empfiehlt sich die Adsorption der Porphyrine an Talk (7). Nach Adsorption der freien Porphyrinsäuren (10 Min.) wird dic Flüssigkeit über eine mit Filterpapier bedeckte Porzellannutsche abgesaugt, wobei sich das Filterpapier mit Talk beschichtet. Dieses wird anschließend bei $35^{\circ}$ etwa $12 \mathrm{Stdn}$. getrocknet und mit Methanol-Schwefelsäure $(5 \% \mathrm{v} / \mathrm{v})$ zur Veresterung der adsorbierten Porphyrine versetzt.

Die Chromatographie erfolgt an DC-Fertigplatten (Schichtdicke $0,25 \mathrm{~mm}$ ) Kieselgel $F_{254}$ (E. Merck) im Lösungsmittelsystem Benzol-Essigsäureäthylester-Methanol (85:13,5:1,5 v/v). Die Tetrapyrrolmethylester werden aus den abgenommenen Kieselgelzonen über Papierfilter mit Chloroform bzw. Chloroform-Methanol (1) eluiert, und das Lösungsmittel wird verdampft. In Kombination mit anderen Lösungsmittelsystemen kann auf demselben Chromatogramm auch Protohäminmethylester herausfraktioniert (8) und durch das Differenzspektrum (reduziert minus oxydiert (10)) bestimmt werden (8).

\section{Spektrophotometrische Analyse}

Kupfer(II)-acteat-Lösungen:

a) Stammlösung $0,2 \%(\mathrm{w} / \mathrm{v}): 400 \mathrm{mg}\left(\mathrm{CH}_{3} \mathrm{COO}\right)_{2} \mathrm{Cu} \cdot \mathrm{H}_{2} \mathrm{O}$ werden in $100 \mathrm{ml}$ Methanol p. a. bei Raumtemperatur gelöst (Magnetrührer) und mit $100 \mathrm{ml} \mathrm{CHCl}_{3}$ p. a. versetzt. Aufbewahrung der Lösung bei $+4^{\circ}$.

b) Gebrauchslösung 0,05\% (w/v): Die Stammlösung wird mit $\mathrm{CHCl}_{3}-\mathrm{CH}_{3} \mathrm{OH}(1: 1 \mathrm{v} / \mathrm{v}) 1: 4$ verdünnt.

Die Porphyrinmethylester werden in der 0,05proz. Kupferacetatlösung sorgfältig gelöst. Die Lösung bleibt zur Bildung der $\mathrm{Cu}$-Chelate mindestens $10 \mathrm{Min}$. bei Raumtemperatur stehen. Die Substanzen werden zwischen 600 und $370 \mathrm{~nm}$ spektrophotometrisch gegen die Kupferacetat-Gebrauchslösung registriert (Abb.). Für Serienbestimmungen reicht die Schreibung ab $500 \mathrm{~nm}$ bis zum jeweiligen Absorptionsmaximum aus. Die Eichkurve von Proto-, Kopro- und Uroporhyrinmethylester-Cu-Chelaten verläuft bis zu einer Extinktion von 1,2 linear. Der Verlauf der Eichkurve über diesen Bereich hinaus wurde nicht geprüft. Zur Berechnung werden millimolare Extinktionskoeffizienten angegeben (Tab.). Die Isolicrung der verwendeten Substanzen

Tab. 1

Absorptionsmaxima und millimolare Extinktionskoeffizienten ( $d=$ $1 \mathrm{~cm}$ ) von Porphyrinmethylester-Cu-Chelaten

Darstellung und Messung in einer Lösung von Kupfer(II)-acetat $0,05 \%(r / v)$ in Chloroform-Methanol $1: 1$

\begin{tabular}{llllll}
\hline Protoporphyrin-IX & $\lambda_{\max }$ & 404 & 530 & 568 & $\mathrm{~nm}$ \\
& $\varepsilon_{\max }$ & 277 & 12,1 & 27,9 & \\
Koprophorphyrin-I(III) & $\lambda_{\max }$ & 397 & 523 & 559 & $\mathrm{~nm}$ \\
& $\varepsilon_{\operatorname{mM}}$ & 381 & 13,3 & 26,8 & \\
Heptacarboxyporphyrin & $\lambda_{\max }$ & 402 & 529 & 567 & $\mathrm{~nm}$ \\
& $\varepsilon_{\operatorname{mM}}$ & 368 & 12,9 & 24,3 & \\
Uroporphyrin-I(III) & $\lambda_{\max }$ & 401 & 526 & 562 & $\mathrm{~nm}$ \\
& $\varepsilon_{\operatorname{mM}}$ & 398 & 12,7 & 24,2 & \\
\hline
\end{tabular}

erfolgte nach 1. c. (1). Der Zeitpunkt der spektrometrischen Messung der Chelatlösung kann den Gegebenheiten angepaßt werden. Bei $+4^{\circ}$ aufbewahrte Lösungen zeigten nach 15 Tagen keine, nach 30 Tagen eine sehr geringe Erhöhung des Extinktion. Qualitative Verschiebungen der Absorption entstanden in diesem Zeitraum nicht. Änderungen in der Konzentration des Kupfer(II)acetats zwischen 0,05 und $0,1 \%$ hatten keinen Einfluß auf die Höhe der millimolaren Extinktionskoeffizienten der Porphyrinmethylester-Cu-Chelate.

Die Arbeit wurde mit Unterstützung der Deutschen Forschungsgemeinschaft durchgeführt.

\section{Literatur}

1. Doss, M., J. Chromatog. 30, 265 (1967). - 2. Doss, M. und W. Mannherm, diese Z. 5, 260 (1967). - 3. Doss, M. und U. Bode, diese Z. 6, 383 (1968). - 4. Doss, M., Dtsch. med. Wschr., in Druck. - 5. Doss, M., Biochim. biophysica Acta, im Druck. 6. Doss, M. und U. BoDE, J. Chromatog., 35, 248 (1968). - 7. FALK, J. E., Porphyrins and Metalloporphyrins, Elsevier, Amsterdam-London-New York (1964). - 8. Doss, M., Klin. Wschr., 46, 731 (1968). - 9. Doss, M. und K. Oetre, diese Z. 3, 125 (1965). - 10. Porra, R. J. und O. T. G. Jones, Biochem. J., 87, 181 (1963).

Dr. Manfred Doss 355 Marburg (Lahn) Pilgrimstein 2

\section{Mehrzweck-Küvette für Immunodiffusionspräparate}

\section{Multipurpose cuvette for immunodiffusion preparations}

\section{Von M. Pérez-Miranda ${ }^{1}$ ) und Hilde Götz}

Aus der Abteilung für klinische Immunologie des.Universitätskrankenbauses Erlangen-Nïrnberg

(Vorstand: Prof. Dr. F. Scheiffartb)

(Eingegangen am 8. August 1968)

Komplette apparative Austüstungen für die heute im klinischen und experimentellen Laboratorium weit verbreiteten Agartechniken, wie Immunodiffusions- und Elektrophoresetechniken auf Objektträgern, stehen hète bereits von namhaften Firmen in verschiedener Ausführung zur Verfügung. Im Zuge der fortschreitenden Automatisierung laboratoriumstechnischer Ver-

1) Stipendiat der Alexander von Humboldt-Stiftung. fahren stellen derartige Angebote eine wahre Bereicherung dar. Es ist jedoch aufgefallen, daß in keinem der entsprechenden Angebote und auch in keiner der uns zugänglich gewordenen Arbeiten über Methodik und technische bzw. apparative Voraussetzungen für Analysen im Agargel, eine Kammer für das schwebesichere Wässern oder für das Eintauchen von Agarplättchen in Inkubationslösungen angegeben ist.

Im folgenden soll deshalb eine Küvette beschrieben werden, die eine zuverlässige Behandlung von Agargelpräparaten auf Objektträgern gestattet. Die Konstruktion dieses u. E. platzsparenden und handlichen Behälters ist so beschaffen, $\mathrm{da} \beta$ vor allem ein Hinwegschwimmen der Agarschichten von den Glasplättchen mit Umkehren der Schichtseite und mit Vermischen der mit Analysen versehenen Agarstreifen mit Sicherheit vermieden wird.

\section{Beschreibung}

Die Küvette besteht aus zwei sich komplementierenden Teilen, einem Gestell und einer eng umschließenden Hülle mit Deckel- 


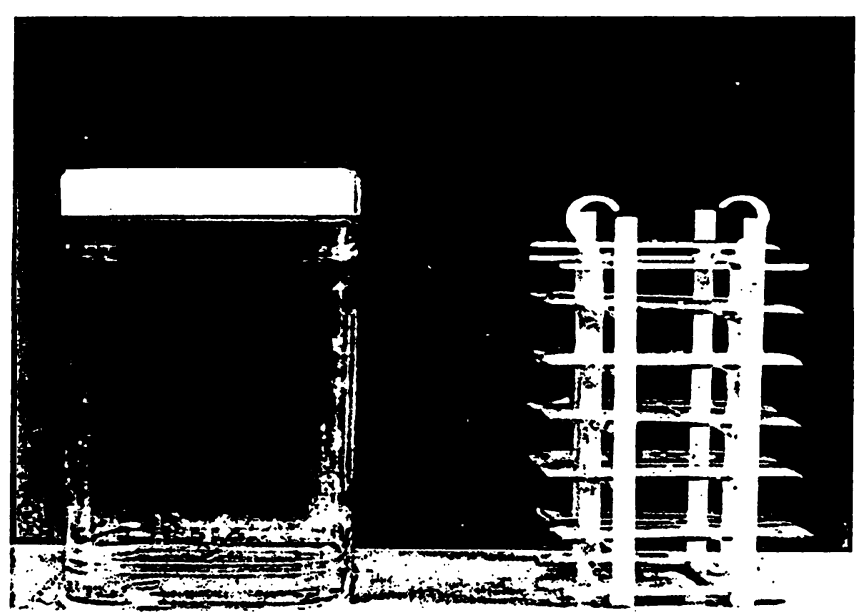

Abb. 1

Gehäuse (li.) und Objektträgergestell (re.) der MehrzweckKüvette
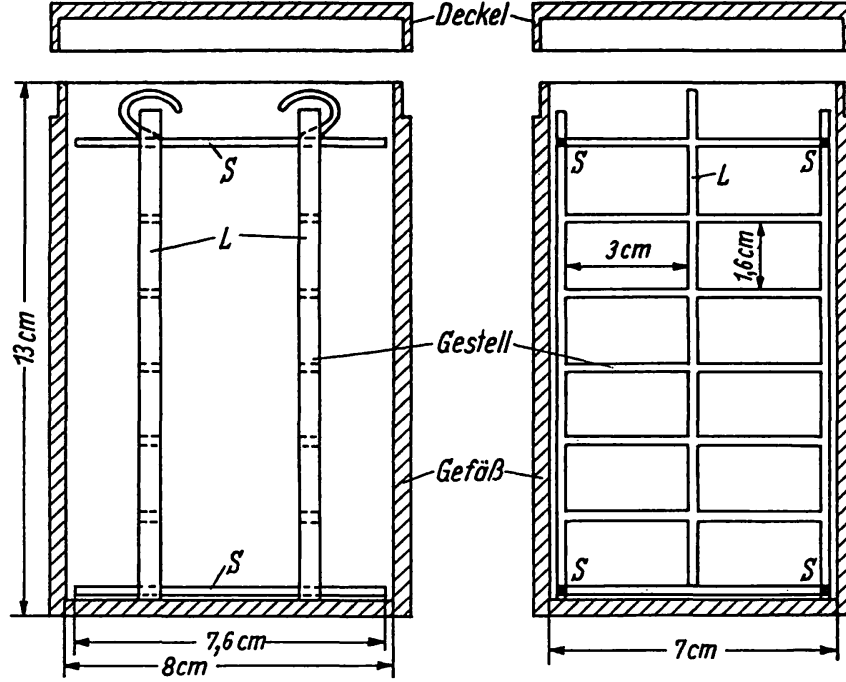

Abb. 2

Technische Angaben zum Aufbau einer Mehrzweck-Küvette für Agartechniken auf Objektträgern

nicht nur als zuverlässiges Gehäuse für alle Wässerungsprozesse von agarbeschickten Objektträgern, sondern sie eignet sich in gleichem Maße auch als Inkubationskammer wie als Kühlraum, wobei die jeweils erforderlichen Temperaturen lediglich über einen Wärme- oder Kühlmantel eingestellt zu werden brauchen. Im einzelnen kommen folgende Verwendungsmöglichkeiten in Frage:

\section{Vervendung als Inkubations- bzw. Feucbtekammer}

Verwendet man die Küvette als Inkubations- bzw. Feuchtekammer, etwa zum Auspräzipitieren von diffundierten Antigen- und Antikörpersystemen, so werden die einzelnen Präparate waagerecht auf die Sprossen der beiden Leiterchen gelegt. Im Falle der noch notwendigen Beschickung der Agaraussparungen bzw. Kanäle (Immunoelektrophorese) kann diese mühelos mit den entsprechenden Lösungen bzw. Antiseren vorgenommen werden (Abb.3). Das Gefäß wird nach den entsprechenden Vorbereitungen mit 10 bis $20 \mathrm{~m} l$ dest. Wasser gefüllt, mit dem Deckel luftdicht verschlossen und entweder bei Zimmertemperatur auf dem Laboratoriumstisch stehen gelassen oder in den Wärmeschrank gebracht.

Die Konstruktion der Mehrzweck-Küvette erlaubt, wie der Name besagen soll, eine vielseitige Verwendbarkeit: sie bewährt sich also

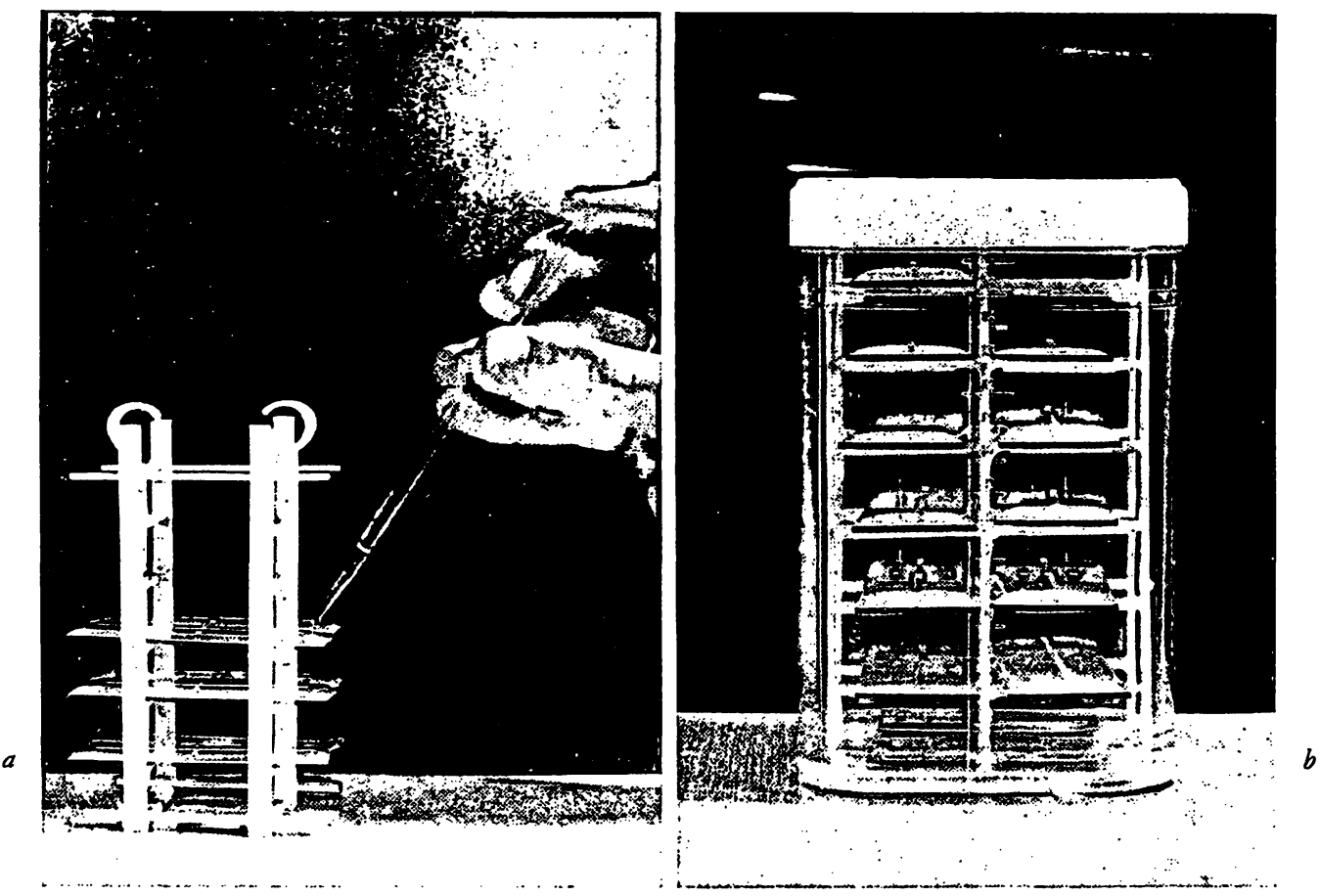

Abb. $3 a$ und $b$

Beschicken der Agarplättchen auf Objektträgern nach deren Auflegen auf die Sprossen des Gestelles einer Mehrzweck-Küvette. Die vorbereiteten Agarplättchen bleiben in gleicher Position (Abb. 3a); nach dem Beschicken wird das Gestell insgesamt in die Küvette (z. B. als feuchte Kammer) eingelassen (Abb. 3b) 
Verwendung als Inkubationskammer mit Substratlösungen fir Fermentnacloweis-Reaktionen

Die Agarplättchen werden nach abgeschlossencr cinfacher Elcktrophorese wicderum auf dic einzelnen Sprossen des Gestelles gelegt, mit dem Gestell in dic Küvettc eingebracht und danach mit der crforderlichen Inkubationslösung beschickt, wobei die Tränkung entweder über doppelte Schichten von Filtrierpapier oder durch Anfüllung der Kammer erfolgt. Dic Küvette kann nunmehr in cine für den Ablauf der Fermentreaktion erwünschtc, konstant zu haltende Umgebungstemperatur gebracht werden. In diesem Falle besteht der Vorteil dieser Küvette darin, daß dic Bewegung des Behälters vom Laboratoriumstisch in den Wärmeschrank bzw. Inkubator und wicder zurück keine Verschiebung der Agarschichten von ihrer Unterlage mit sich bringt.

\section{Verwendung der Kilvette zur IVässerung bzu. Entfärbung oder Fixierung} von Fraktionen in Agarnilieu

Hierfür eignet sich die Kammer insofern in ganz besonderem $\mathrm{Maße}$, als sie ein mehrfaches Wechseln durch einfaches Herausnehmen des gesamten Gestelles aus der Küvettc gestattet ohne Verschiebung der aufgelegten Agarplättchen und wiederum ohne cin Hinwegschwimmen der Agarschichten von den Objektträgern. Sic erlaubt sogar einen leichten Durchfluß von Spülflüssigkeit oder Entfärbelösungen, wodurch zugleich dic cinzelnen Arbeitsgänge wesentlich verkürzt werden können.

\section{Vorwendung der MelJrzweck-Kinvette als Färbebad}

In gleicher Weise kann die Küvette als Färbebad Verwendung finden, wobei hier neben den bereits crwähnten allgemeinen
Sicherheitsfaktoren für das Haftenbleiben der Agarschichten an ihren Objektträgern der Vorteil darin bestcht, daß im Vergleich zu offenen Küvetten der Verdunstungseffekt der Färbclösung weitgehend herabgesetzt werden kann. Darüber hinaus können Färbclösungen unter Bcrücksichtigung der Tatsache, daß dic Küvette belicbig hoch angefüllt werden kann, rationcll aufgebraucht werden. Es sei schlicßlich darauf hingewiesen, daß selbstverständlich dic Küvettc für cine Viclzahl aufcinanderfolgender Vorgänge gecignet ist, ohne daß dic beladenen Gestelle in ihter Anordnung verändert zu werden brauchen.

\section{Besprechung der Konstruktion}

Die wesentlichen Vorteile der Küvette sind bereits im vorangegangenen Abschnitt über dic Verwendungsmöglichkciten erläutert worden. Es sei hier in Kürze auf einige andere, vorwicgend technische, Vorteile hingewiesen.

Die Küvette besteht aus transparentem Material. Ihr Hochformat bei kleinem Grundriß nimmt wenig Platz am Laboratoriumstisch bzw. im Inkubator ein. Ihre vielfältige Anwendbarkeit, möglicherweise sogar im Verlaufe eines Arbeitsganges, gestattet ein rationelles Verarbeiten der agarelcktrophoretisch oder im Rahmen der Immunodiffusionstechniken angesetzten Analysen. Sie erscheint uns deshalb unentbehrlich in einer modernen Ausrüstung für Immunoclektrophorese und Immunodiffusionstechniken.

\section{Dr. M. Pérez-Miranda und}

Priv. Doz. Dr. Hilde Götz

8520 Erlangen, Krankenhausstraße 12

\section{Die Eisenbestimmung in frischen und gelagerten Seren}

\section{Determination of iron in fresh and stored sera}

Von K. Bonitz und G. RogGe

Aus den Zentrallaboratorium (Chefarzt: Dr. K. Bonitz) des Allg. Krankenbauses Hamburg-Eilbek

(Ärztlicher Direletor: Prof. Dr. P. Laurentius)

(Eingegangen am 14. August 1968)

Die ersten Arbeiten über einen Gehalt des Serums an Eisen, welches nicht aus dem Hämoglobin stammt, gehen auf BARKAN zurück $(1,2)$. Der größte Teil dieses Eisens ist an die Serumeiweißfraktion IV-7, die hauptsächlich aus $\beta_{1}$-Globulinen bestcht, gebunden. Ublicherweise bezeichnet man dieses Eisenträgerprotein Siderophilin oder Transferrin $(3,4)$.

Die meisten Bestimmungsmethoden benützen zur Spaltung dieses Eisen-Eiweiß-Komplexes Salzsäure, wobei die Reaktionszeit von der Säurekonzentration abhängig ist. Insbesondere bei Seren, die längere Zeit aufbewahrt wurden, können nach Behandlung mit verdünnter Salzsäure mitunter nur $75-80 \%$ des Serumcisens vom Eiweiß abgespalten werden (5). Auch eine Behandlung mit Trichloressigsäure allein bei Raumtempcratur genügt nicht zur vollständigen Freisetzung (6).

Der nächste Schritt der Bestimmung ist bei vielen Modifikationen die Abtrennung des Eiweißes. Eine Fällung mit Trichloressigsäurc birgt mehrere Fehlerquellen in sich: Eisen kann mit in den Niederschlag gerissen werden $(7,8)$, oder es wird kein optisch klares Filtrat erzielt (9), oder es werden durch dic Vielfalt der Einzeloperationen Eisenspuren cingeschleppt.

In letztcr Zeit wurden einige Methoden beschricben, die eine Eiweißfällung vermeiden und das Scrumeisen dirckt mit einem Farbreagenz bestimmen.

Durch Zugabe eines Detergens kann Eisen vom Eiweiß abgelöst werden und Farbkomplex sowie Eiweiß in Lösung gehalten werden (10-12). Dic Auswahl des gecigneten Detergens und die Abhängigkeit von wechselnden Qualitäten einzclner Chargen ist kritisch $(13,14)$. Ein bestimmtes Mengenverhältnis Serum zu Detergens muß cingchalten werden, die Einwirkungsdauer darf nicht zu kurz sein, mitunter treten Eiweißfällungen auf oder das im Detcrgens gelöstc Reduktionsmittel neigt zur Zersetzung $(15,16)$.

Ebenfalls in letztcr Zcit wurden Methoden bekannt, bei welchen Eisen in cinem bestimmten $\mathrm{pH}$-Bereich vom Eiweiß abgespalten wird und ohne Entciwcißung direkt mit einer Farbreaktion bestimmt wird. Verwendet wird z. B. cin Acetatpuffer von $\mathrm{pH}$ 4,5-5,5; die Ubereinstimmung mit Werten in enteiweißtem Serum ist gut (17-19). A. L. SCHADE und Mitarbeiter fanden, daß in 0,2M Phosphatpuffer $\mathrm{pH}$ 6,8 der Eisen-Siderophilin-Komplex vollständig dissoziiert $(20-22)$. Je niedriger das $\mathrm{pH}$ und je konzentrierter der Puffer, um so sicherer wird das Eisen abgespalten. D. E. Schwartz hat auf diesem Prinzip eine Methode aufgebaut, bei der das freigesetzte Eisen mit Ascorbinsäure reduziert und dann mit sulfoniertem Bathophenanthrolin zu einem Farbkomplex umgesetzt wird, der photometrisch bestimmt werden kann $(23,24)$. Für die Durchführung der Eisenbestimmung nach dieser Methode sind gebrauchsfertige Reagenziensätze im Handel.

In jüngster Zeit wurde mitgeteilt, daß bei älteren Seren die Bestimmung des Eisens ohne vorhergehende Enteiweißung infolge Auftretens von Trübungen beim Pufferzusatz gestört wird, während mit frischen Seren die mit verschiedenen Methoden mit und ohne Enteiweißung gewonnenen Ergebnisse gut übereinstimmen (25).

Um festzustellen, wie oft im Durchschnitt mit Störungen bei der Analysc von gelagerten Seren zu rechnen ist, wurde Eisen ohne Enteiweißung jeweils in frischem Scrum, nach 4 Tagen und nach 8 Tagen bestimmt (Mcrckotest Eiscn, Art. Nr. 3307).

Die durchschnittliche Strcuung der Werte (Präzision von Tag zu Tag) als Standardabweichung ausgedrückt, betrug $\pm 3,0 \mu \mathrm{g}$ Eiscn pro $100 \mathrm{~m} /$. Von 100 Seren zeigten am 4 . bzw. 5 . Tag gegenüber dem Ausgangswert 9 Seren cine signifikante (größcr als $\pm 5 \%$ ) Abweichung, davon waren 7 Werte erhöht und 2 Werte erniedrigt. Am 8. Tag waren 12 Seren signifikant verändert, davon 9 erhüht 
Tab. 1

Eisenbestimmung in gelagerten Seren

\begin{tabular}{|c|c|c|c|c|c|c|c|c|c|}
\hline \multirow{2}{*}{$\begin{array}{c}\text { Serum } \\
\text { Nr. }\end{array}$} & \multirow{2}{*}{$\begin{array}{l}\text { 1. Tag } \\
\mu \mathrm{g} \mathrm{Fe/} \\
100 \mathrm{ml}\end{array}$} & \multicolumn{2}{|c|}{ 4. bzw. 5. Tag } & \multirow{2}{*}{$\Delta \%$} & \multirow{2}{*}{\multicolumn{2}{|c|}{$\begin{array}{l}\text { 8. Tag } \\
\Delta \underset{\mathrm{Fe} /}{\mu \mathrm{Fe}} \mathrm{ml}\end{array}$}} & \multirow[b]{2}{*}{$\Delta \%$} & \multirow[b]{2}{*}{ $\pm s$} & \multirow[b]{2}{*}{ $\pm s \%$} \\
\hline & & $\underset{100 \mathrm{ml}}{\mu \mathrm{Fe} /}$ & ${ }_{100 \mathrm{ml}} \mathrm{Fe}^{-}$ & & & & & & \\
\hline $\begin{array}{l}36 \\
39 \\
41 \\
48 \\
56 \\
63 \\
64 \\
66 \\
69 \\
71 \\
73 \\
80 \\
82 \\
89 \\
97\end{array}$ & $\begin{array}{r}31 \\
294 \\
88 \\
96 \\
68 \\
156 \\
32 \\
31 \\
63 \\
74 \\
184 \\
47 \\
82 \\
32 \\
152\end{array}$ & $\begin{array}{r}32 \\
301 \\
82 \\
120 \\
86 \\
167 \\
30 \\
31 \\
75 \\
73 \\
193 \\
48 \\
87 \\
42 \\
167\end{array}$ & $\begin{array}{l}+1 \\
+7 \\
+6 \\
+24 \\
+18 \\
+11 \\
-2 \\
0 \\
+12 \\
-1 \\
+9 \\
+1 \\
+5 \\
+10 \\
+15\end{array}$ & $\begin{array}{r}3,2 \\
2,4 \\
-7,5 \\
20,0 \\
26,5 \\
7,1 \\
-6,2 \\
0 \\
19,0 \\
1,4 \\
4,9 \\
2,1 \\
6,1 \\
31,2 \\
9,9\end{array}$ & $\begin{array}{r}40 \\
309 \\
70 \\
101 \\
85 \\
162 \\
18 \\
42 \\
65 \\
82 \\
209 \\
34 \\
95 \\
40 \\
173\end{array}$ & $\begin{array}{l}+9 \\
+15 \\
+18 \\
+5 \\
+17 \\
+6 \\
+14 \\
+11 \\
+2 \\
+8 \\
+25 \\
+13 \\
+13 \\
+8 \\
+21\end{array}$ & $\begin{array}{r}22,5 \\
5,1 \\
-25,7 \\
5,0 \\
25,0 \\
3,8 \\
-43,7 \\
26,2 \\
3,2 \\
9,8 \\
13,6 \\
-27,7 \\
15,9 \\
20,0 \\
13,8\end{array}$ & $\begin{array}{r}4,9 \\
7,5 \\
9,2 \\
12,7 \\
10,1 \\
5,5 \\
7,6 \\
6,4 \\
6,4 \\
4,9 \\
12,7 \\
7,8 \\
6,6 \\
5,3 \\
10,8\end{array}$ & $\begin{array}{r}14,4 \\
2,5 \\
11,5 \\
12,0 \\
12,7 \\
3,4 \\
28,4 \\
18,3 \\
9,5 \\
6,5 \\
6,5 \\
18,2 \\
7,5 \\
13,9 \\
6,6\end{array}$ \\
\hline
\end{tabular}

und 3 erniedrigt. 3 Seren zeigten am 4. Tag einen Anstieg und waren am 8. Tag wieder abgefallen. Alle weiteren Einzelheiten können aus der Tabelle 1 entnommen werden.

Es zeigte sich damit, daß nur bei einem getingen Prozentsatz der auf Eisen untersuchten älteren Seren eine Erhöhung des Meßwertes zu erwarten ist. Unserer Meinung nach ist es für ein Routinelaboratorium nicht $z u$ vertreten. $\mathrm{da} B$ auf die vereinfachte Ausfühgung der Eisenbestimmung verzichtet wird, nur um auch bei längere Zeit gelagerten Seren diejenigen (größenordnungsmäßig $10 \%$ ) richtig $\mathrm{zu}$ bestimmen, in welchem andernfalls um bis $\mathrm{zu}$ $25 \mu \mathrm{g}$ pro $100 \mathrm{ml}$ (absolut) zu hohe Eisenwerte erhalten werden.

\section{Literatur}

1. Barkan, G., Hoppe-Seyler's Z. physiol. Chem. 148, 124 (1925), 171, 194 (1927), 216, 1 (1933). - 2. Barkan, G. und O. Schales, Hoppe-Seyler's Z. physiol. Chem. 248, 96 (1937). - 3. Edsall, J. T., Adv. in Prot. Chem. 3, 383 (1947). - 4. Ramsay, W. N. M. in H. Sobotka und C. P. Stewart (Ed.) Adv. in Clin. Chem. 1, 1 (1958). - 5. Peters T., Th. Giovaniello, L. Apt und J. Ross, J. Laborat. Clin. Med. S. Louis 48, 280 (1956). - 6. KINGSLEY, G. R. und G. Getchell, Clin. Chem. New Yoik 2, 175 (1956). - 7. Bothwell, T. H. und B. Malletr, Biochem. J. 59, 599 (1955). - 8. Ramsay, W. N. M., Biochem. J.
53, 227 (1953). - 9. Henry, R. J., C. Sobel und N. ChiaMORI, Clin. chimica Acta Amsterdam 3, 523, (1958). - 10. Lauber, K., diese Z. 3, 96 (1965). - 11. Sanford, R., J. Clin. Path. London 16, 174 (1963). - 12. Webster, D., J. Clin. Path. London 13, 246 (1960). - 13. Dronskr, H. und G. Galts, Dtsch. Ges.wesen 21, 2457 (1966). — 14. Hoppe, F., Fortschr. Med. 84, 283 (1966). - 15. Askevold, R. und O. D. Vellar, Scand. J. clin. Lab. Invest. 20, 122 (1967). - 16. Sснмrdt, G., Dtsch. Ges.wesen 22, 1686 (1967). - 17. Brownstern, H., Amer. J. Clin. Path. 47, 714 (1967). - 18. Goodwin, J. F., B. Murphy und M. Guillemerta, Clin. Chem. New York 12, 47 (1966). 19. Ness, A. T. und H. G. Dickerson, Clin. chimica Acta Amsterdam 12, 579 (1965). - 20. Levy, A. C. und P. VitracCA, Clin. Chem. New York 7, 241 (1961). - 21. Nelson, C. W. Amer. J. Med. Techn. 30, 71 (1964). - 22. Schade, A. L., J. Oyama, R. W. Reinhart und I. R. Miller, Proc. Soc. Exper. Biol. Med. 87, 443 (1954). - 23. Fürr, J., Med. Monatsschr. 19, 281 (1965). - 24. Schwartz, D. E., Bull. der Schweiz. Vereinigung f. klin. Chemie, 1957, Nt. 1. - 25. HoeflmaYer, J. und R. FrIED, Med. Klinik 61, 1820 (1966).

Dr. K. Bonitz
2 Hamburg 22
Friedrichsberger Str. 60

\section{MITTEILUNGEN}

\section{Deutsche Gesellschaft für Hämatologie}

Auf der 13. Tagung der Deutschen Gesellschaft für Hämatologie in Ulm/Donau wurde Prof. Dr. K. LeNNERT (Kiel) zum neuen Kongreß-Präsidenten für 1969 gewählt. Die nächste Tagung der Gesellschaft wird in der ersten Septemberwoche 1969 in Kiel stattfinden.

Prof. Dr. W. Sтrсн (München) wurde für die Zeit von 1968-1970 zum Vorsitzenden der Gesellschaft wiedergewählt.

Dr. K. G. von BoroviczenY (Freiburg/Breisgau) wurde zum neuen Sekretär der Gesellschaft gewählt.

Auf der Tagung wurde außerdem ein Ausschuß für die Vorbereitung des XIII. Kongresses der Internationalen Gesellschaft für Hämatologie gebildet, der 1970 in München unter dem Präsidium von Prof. Dr. L. Heilmeyer (Ulm) stattfinden wird.
Es wird auf die Neugründung der

\section{Europäischen Vereinigung für Krebsforschung}

(European Association for Cancer Research) aufmerksam gemacht.

Zweck dieser Vereinbarung ist es, alle zwei Jahre einen wissenschaftlichen Krebskongreß abzuhalten; der erste wird im Oktober 1969 in der Schweiz stattfinden.

Anmeldeformulare und weitere Informationen k̦önnen vom Sekretär der Vereinigung, Herrn Dr. G. J. V. SWAEN, Academisch Ziekenhuis Wilhelmina Gasthuis, Pathologisch-Anatomisch Laboratorium, Amsterdam-Oud West, Eerste Helmersstraat 104, Niederlande, erhalten werden. 